\title{
Hyoscine butylbromide versus acetaminophen for nonspecific colicky abdominal pain in children: a randomized controlled trial
}

\author{
Naveen Poonai MD, Kriti Kumar MD, Kamary Coriolano PhD, Graham Thompson MD, Shaily Brahmbhatt BSc, \\ Emily Dzongowski BSc, Holly Stevens BSc, Priti Gupta BSc, Michael Miller PhD, Sharlene Elsie, \\ Dhandapani Ashok MD, Gary Joubert MD, Rod Lim MD, Andreana Bütter MD, Samina Ali MDCM
}

Cite as: CMAJ 2020 November 30;192:E1612-9. doi: 10.1503/cmaj.201055

See related commentary at www.cmaj.ca/lookup/doi/10.1503/cmaj.202555

\begin{abstract}
BACKGROUND: Less than two-thirds of children with abdominal pain in the emergency department receive analgesia. We sought to determine whether hyoscine butylbromide was superior to acetaminophen for children with nonspecific colicky abdominal pain.
\end{abstract}

METHODS: We randomly allocated children aged 8-17 years with nonspecific colicky abdominal pain who presented to the pediatric emergency department of London Health Sciences Centre, London, Ontario to receive hyoscine butylbromide, $10 \mathrm{mg}$ given orally, or acetaminophen, $15 \mathrm{mg} / \mathrm{kg}$ given orally (maximum $975 \mathrm{mg}$ ). We considered the minimal clinically important difference for the primary outcome (self-reported pain at $80 \mathrm{~min}$ ) to be $13 \mathrm{~mm}$ on a $100 \mathrm{~mm}$ visual analogue scale. Secondary outcomes included administration of rescue analgesia, adverse effects and pain score less than $30 \mathrm{~mm}$ at 80 minutes.

RESULTS: A total of 236 participants (120 in the hyoscine butylbromide group and 116 in the acetaminophen group) were included in the trial. The mean visual analogue scale scores at 80 minutes were $29 \mathrm{~mm}$ (standard deviation [SD] $26 \mathrm{~mm}$ ) and $30 \mathrm{~mm}$ (SD $29 \mathrm{~mm}$ ) with hyoscine butylbromide and acetaminophen, respectively (adjusted difference 1, 95\% confidence interval -7 to 7 ). Rescue analgesia was administered to 4 participants (3.3\%) in the hyoscine butylbromide group and 1 participant $(0.9 \%)$ in the acetaminophen groups $(p=0.2)$. We found no significant differences in rates of adverse effects between hyoscine butylbromide (32/116 [27.6\%]) and acetaminophen (28/115 [24.3]) $(p=$ $0.5)$; no serious adverse effects were observed. The proportion with a pain score less than $30 \mathrm{~mm}$ at 80 minutes was 66 (55.0\%) with hyoscine butylbromide and 63 (54.3\%) with acetaminophen $(p=0.9)$.

INTERPRETATION: Hyoscine butylbromide was not superior to acetaminophen in this setting. Both agents were associated with clinically important pain reduction, and either can be considered for children presenting to the emergency department with nonspecific colicky abdominal pain. Trial registration: Clinicaltrials.gov, no. NCT02582307
A

bdominal pain is reported by a third of school-aged children $^{1}$ and accounts for several visits daily in most emergency departments. ${ }^{2-5}$ Although the use of analgesia to treat acute abdominal pain is well-supported, ${ }^{6,7}$ there is little evidence to guide the management of nonspecific abdominal pain in the emergency department, ${ }^{8}$ which accounts for two-thirds of cases of abdominal pain presenting to the emergency department., Acetaminophen is the most commonly used World Health Organization Step 1 analgesic. ${ }^{10}$ In children, it is effective for many painful conditions, ${ }^{11,12}$ but data supporting its use for abdominal pain are lacking. ${ }^{13,14}$ Despite strong advocacy by the American Academy of Pediatrics ${ }^{15}$ for adequate pain management, less than two-thirds of children with abdominal pain in the emergency department receive analgesia, ${ }^{16,17}$ and roughly half experience ongoing pain after discharge. ${ }^{18}$ Children with nonspecific abdominal pain are less likely than those with a specific cause to receive analgesia. ${ }^{5}$ Available analgesic options for children with nonspecific abdominal pain in the emergency department may result in greater adherence to the American Academy of Pediatrics recommendations.

Hyoscine butylbromide is orally administered and available in most Canadian emergency departments. We surmised that it may be effective for colicky abdominal pain owing to its antispasmodic properties. ${ }^{19}$ Ten placebo-controlled studies involving 3699 adults with functional abdominal pain showed hyoscine butylbromide to be beneficial, without serious adverse effects. ${ }^{20-29}$ In the only pediatric study, hyoscine butylbromide, $10 \mathrm{mg}$ given orally, was found 
to be beneficial compared to a homeopathic preparation in 204 children, with no serious adverse effects. ${ }^{30}$ We sought to determine whether hyoscine butylbromide was superior to acetaminophen in relieving pain among children presenting to the emergency department with nonspecific colicky abdominal pain.

\section{Methods}

\section{Study design and setting}

We conducted a double-blind randomized trial to test the hypothesis that hyoscine butylbromide is superior to acetaminophen for children with nonspecific colicky abdominal pain. Research assistants (K.K., S.B., S.E., E.D.) screened consecutive potentially eligible participants in the pediatric emergency department of London Health Sciences Centre, London, Ontario, daily between the hours of 1700 and 2400 from Mar. 20, 2017, to Dec. 3, 2018. The emergency department has an annual census of 38000 visits and is the only pediatric surgical referral centre in southwestern Ontario.

\section{Participant selection}

We included children aged 8-17 years with abdominal pain selfreported as "crampy," "coming in waves" or "squeezing," and rated as $40 \mathrm{~mm}$ on a $100 \mathrm{~mm}$ visual analogue scale ${ }^{31}$ at its least intense. Pain was assessed immediately before enrolment. Children were excluded if they were unable to swallow pills or communicate verbally, currently used an anticholinergic, had hypersensitivity to acetaminophen, hyoscine butylbromide or applesauce vehicle, had received acetaminophen or hyoscine butylbromide within 6 hours of enrolment, had incurred abdominal trauma within 48 hours of enrolment or had medical record evidence of abdominal or genitourinary disease; those in whom a surgical or medical cause for the pain (e.g., appendicitis, renal colic, bowel obstruction) was suspected clinically or radiographically were also excluded. The full study protocol is provided in Appendix 1 (available at www.cmaj.ca/lookup/doi/10.1503/ cmaj.201055/tab-related-content). We used a 5-member focus group of caregivers of children with abdominal pain to inform the terminology for describing colicky abdominal pain, lower age limit for swallowing pills, and consent and assent forms.

Participants were randomly allocated in a 1:1 allocation ratio with permuted block sizes to either single-dose hyoscine butylbromide in tablet form, $10 \mathrm{mg}$ given orally (Boehringer Ingelheim), ${ }^{30}$ plus placebo acetaminophen liquid (Perrigo); or acetaminophen liquid, $15 \mathrm{mg} / \mathrm{kg}$ given orally to a maximum of $975 \mathrm{mg}$ (McNeil Consumer Healthcare) plus placebo hyoscine butylbromide tablet (Perrigo). Preparation of medications, allocation concealment and implementation of randomization were pharmacy controlled. The randomization list was generated with a computer-based random-number generator (www. randomization.com). Allocation concealment was performed by means of sequentially numbered, opaque, sealed envelopes.

Medications were administered by the bedside nurse. If the patient vomited within 30 minutes of receiving the medication, another dose was given. Rescue analgesia was permitted at any time. Blinded parties included the participant, caregiver, emergency department personnel and all members of the study team apart from the pharmacist.

\section{Outcomes}

Outcome data were collected by research assistants (K.K., S.B., S.E., E.D.) using an iPad hosting the Research Electronic Data Capture (REDCap) platform. ${ }^{32}$ The primary outcome was self-reported pain 80 minutes after the intervention, assessed with a $100 \mathrm{~mm}$ visual analogue scale. ${ }^{33}$ Eighty minutes reflects the time to peak analgesic action of hyoscine butylbromide ${ }^{19}$ and acetaminophen (60-90 min). ${ }^{34,35}$ The visual analogue scale has been used in trials of analgesics in children older than 6 years of age $\mathrm{e}^{36,37}$ and there are abundant data establishing its reliability. ${ }^{38-43}$ Secondary outcomes included rescue analgesia, adverse effects and a visual analogue scale pain score less than $30 \mathrm{~mm}$ after the intervention, the World Health Organization target for effective analgesia. ${ }^{44}$ The following adverse effects were considered serious: hospital admission due to a drug-related event, prolongation of existing hospital stay, persistent or major disability or incapacity, a life-threatening outcome and death. Other secondary outcomes included caregiver satisfaction with pain management, assessed with a Likert scale ranging from 1 (very unsatisfied) to 5 (very satisfied), selfreported pain scores 15, 30, 45 and 60 minutes after the intervention, return visits to a health care provider, missed surgical diagnoses within 72 hours of emergency department discharge, emergency department length of stay, discharge diagnosis, disposition and time to a $20 \%$ reduction in preintervention pain (Appendix 2, available at www.cmaj.ca/lookup/doi/10.1503/cmaj.201055/tab-related-content). All outcomes were prespecified except pain after discharge, length of emergency department stay and missed surgical diagnoses (data collected by means of a telephone survey with caregivers at $72 \mathrm{~h}$, as detailed in Appendix 2).

\section{Deviations from registered protocol}

Deviations from the registered protocol are detailed in Appendix 1. The most significant deviation was that only the visual analogue scale was used to determine eligibility and assess pain because it has established reliability when used with a tablet device ${ }^{38}$ and we believed it to be more acceptable to the age of the participants.

\section{Sample size}

We used a minimal clinically important difference on the visual analogue scale of $13 \mathrm{~mm}$ between groups based on a derivation cohort ${ }^{45}$ and a validation cohort, ${ }^{39}$ and an adult emergency department study of hyoscine butylbromide and acetaminophen for abdominal pain. ${ }^{46}$ With a standard deviation (SD) of $30 \mathrm{~mm}$, 112 children per group were required to detect a difference at the $5 \% 2$-sided level of significance with $90 \%$ power. The sample size was increased to account for dropouts, giving a final sample size of 115 participants per group.

\section{Statistical analysis}

Analyses of efficacy outcomes were based on intention to treat. In participants without an 80-minute visual analogue scale score, we assumed that the score was unchanged from the preintervention pain score. Analysis of adverse effects and of caregiver satisfaction was based on a per protocol analysis. We performed inferential statistics on primary and secondary efficacy outcomes. We used means and SDs, frequencies and percentages, and medians and interquartile ranges (IQRs) to summarize ratio, categoric and ordinal data, 
respectively. We compared pain scores at 80 minutes between groups using linear regression, adjusting for pain score immediately before the intervention. We reported time to achieve at least a $20 \%$ reduction in preintervention pain using a Kaplan-Meier survival analysis. We compared categoric variables using the Pearson $\chi^{2}$ test and adverse events using the Fisher exact test. Post hoc regression analyses explored the effect of analgesia provided more than 6 hours before the intervention on the primary outcome with a test of interaction. We analyzed the data using SPSS version 24 (IBM Corp.). We considered $p$ values less than 0.05 statistically significant.

\section{Ethics approval}

The protocol received approval from Western University's Health Sciences Research Ethics Board. The committee would not approve the use of a placebo. The trial was monitored by an independent data safety monitoring board.

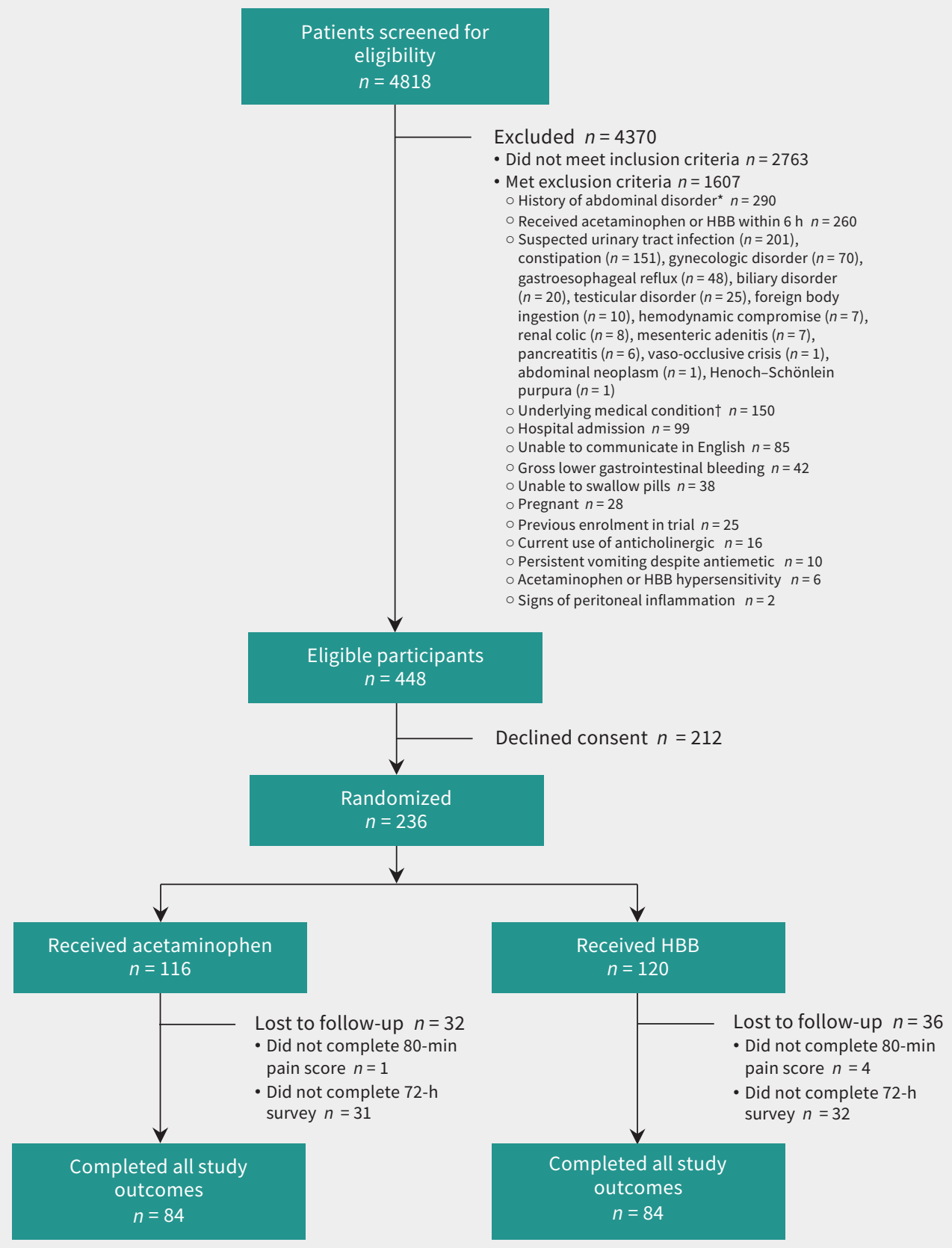

Figure 1: Flow diagram showing participant selection. *History of abdominal disorder included abdominal surgery $(n=167)$, abdominal trauma within 48 hours $(n=42)$, cyclic vomiting $(n=24)$, celiac disease $(n=18)$, hepatobiliary disease $(n=14)$, bowel obstruction $(n=13)$ and chromosomal abnormality affecting abdominal viscera $(n=12)$. †Underlying medical conditions included congenital renal anomaly $(n=55)$, congenital genitourinary anomaly $(n=53)$, inflammatory bowel disease $(n=37)$, pelvic inflammatory disease $(n=3)$, neutropenia $(n=1)$ and tuberculosis $(n=1)$. Note: HBB $=$ hyoscine butylbromide. 
Table 1 (part 1 of 2): Baseline characteristics of children with nonspecific colicky abdominal pain randomized to receive hyosine butylbromide or acetaminophen

\section{No. $(\%)$ of patients*}

\begin{tabular}{|c|c|c|}
\hline Characteristic & $\begin{array}{l}\text { Hyoscine } \\
\text { butylbromide } \\
n=120\end{array}$ & $\begin{array}{l}\text { Acetaminophen } \\
\qquad n=116\end{array}$ \\
\hline Female sex & $79(65.8)$ & $74(63.8)$ \\
\hline Age, mean $\pm S D$, yr & $12.5 \pm 3.1$ & $12.4 \pm 2.9$ \\
\hline $\begin{array}{l}\text { Duration of pain before } \\
\text { enrolment, median (IQR), } \mathrm{h}\end{array}$ & 2 (0.5 to 5$)$ & 1.8 (0.5 to 4.8$)$ \\
\hline \multicolumn{3}{|l|}{$\begin{array}{l}\text { Received analgesia before } \\
\text { enrolment } \dagger\end{array}$} \\
\hline Acetaminophen & $13(10.8)$ & $9(7.8)$ \\
\hline Ibuprofen & $20(16.7)$ & $24(20.7)$ \\
\hline Ketorolac & $2(1.7)$ & $4(3.4)$ \\
\hline $\begin{array}{l}\text { Received antiemetic in } \\
\text { emergency department before } \\
\text { enrolment } \ddagger\end{array}$ & $28(23.3)$ & $23(19.8)$ \\
\hline $\begin{array}{l}\text { Received antacid in emergency } \\
\text { department before enrolment§ }\end{array}$ & $2(1.7)$ & $2(1.7)$ \\
\hline \multicolumn{3}{|l|}{ Comorbiditiesף } \\
\hline None & $70(58.3)$ & $72(62.1)$ \\
\hline Gastrointestinal & $13(10.8)$ & $12(10.3)$ \\
\hline Gastritis & $1(0.8)$ & $2(1.7)$ \\
\hline Functional abdominal pain & $8(6.7)$ & $6(5.2)$ \\
\hline Irritable bowel syndrome & $1(0.8)$ & $1(0.9)$ \\
\hline $\begin{array}{l}\text { Recurrent abdominal pain of } \\
\text { childhood }\end{array}$ & $0(0.0)$ & $2(1.7)$ \\
\hline Gastroesophageal reflux & $2(1.7)$ & $1(0.9)$ \\
\hline Functional dyspepsia & $1(0.8)$ & $0(0.0)$ \\
\hline Psychiatric & $24(20.0)$ & $15(12.9)$ \\
\hline Anxiety & $10(8.3)$ & $10(8.6)$ \\
\hline Depression & $3(2.5)$ & $3(2.6)$ \\
\hline Posttraumatic stress disorder & $5(4.2)$ & $0(0.0)$ \\
\hline Learning disability & $1(0.8)$ & $0(0.0)$ \\
\hline Eating disorder & $0(0.0)$ & $1(0.9)$ \\
\hline $\begin{array}{l}\text { Attention deficit hyperactivity } \\
\text { disorder }\end{array}$ & $5(4.2)$ & $1(0.9)$ \\
\hline Otolaryngologic & $9(7.5)$ & $9(7.8)$ \\
\hline Recurrent otitis media & $2(1.7)$ & $4(3.4)$ \\
\hline Obstructive sleep apnea & $1(0.8)$ & $0(0.0)$ \\
\hline Environmental allergies & $2(1.7)$ & $3(2.6)$ \\
\hline Recurrent tonsillitis & $2(1.7)$ & $2(1.7)$ \\
\hline Hearing impairment & $1(0.8)$ & $0(0.0)$ \\
\hline Branchial cleft cyst & $1(0.8)$ & $0(0.0)$ \\
\hline Urologic & $2(1.7)$ & $3(2.6)$ \\
\hline $\begin{array}{l}\text { Recurrent urinary tract } \\
\text { infections }\end{array}$ & $2(1.7)$ & $2(1.7)$ \\
\hline IgA nephropathy & $0(0.0)$ & $1(0.9)$ \\
\hline
\end{tabular}

Table 1 (part 2 of 2): Baseline characteristics of children with nonspecific colicky abdominal pain randomized to receive hyosine butylbromide or acetaminophen

\section{No. $(\%)$ of patients*}

\begin{tabular}{|c|c|c|}
\hline Characteristic & $\begin{array}{c}\text { Hyoscine } \\
\text { butylbromide } \\
n=120\end{array}$ & $\begin{array}{l}\text { Acetaminophen } \\
\qquad n=116\end{array}$ \\
\hline Orthopedic & $4(3.3)$ & $1(0.9)$ \\
\hline In-toeing & $2(1.7)$ & $1(0.9)$ \\
\hline Scoliosis & $1(0.8)$ & $0(0.0)$ \\
\hline Osteochondritis dessecans & $1(0.8)$ & $0(0.0)$ \\
\hline Endocrinologic & $4(3.3)$ & $2(1.7)$ \\
\hline Turner syndrome & $1(0.8)$ & $1(0.9)$ \\
\hline Short stature & $1(0.8)$ & $1(0.9)$ \\
\hline Menorrhagia & $1(0.8)$ & $0(0.0)$ \\
\hline Type I diabetes mellitus & $1(0.8)$ & $0(0.0)$ \\
\hline Ophthalmologic & $0(0.0)$ & $3(2.6)$ \\
\hline Strabismus & $0(0.0)$ & $2(1.7)$ \\
\hline Carotid ophthalmic aneurysm & $0(0.0)$ & $1(0.9)$ \\
\hline Neurologic: migraine & $2(1.7)$ & $1(0.9)$ \\
\hline Respiratory: asthma & $5(4.2)$ & $2(1.7)$ \\
\hline Hematologic: thalassemia & $0(0.0)$ & $1(0.9)$ \\
\hline Other & $0(0.0)$ & $3(2.6)$ \\
\hline Fibromyalgia & $0(0.0)$ & $1(0.9)$ \\
\hline Chronic fatigue & $0(0.0)$ & $1(0.9)$ \\
\hline Maple syrup urine disease & $0(0.0)$ & $1(0.9)$ \\
\hline
\end{tabular}

Note: IgA = immunoglobulin A, IQR = interquartile range, $\mathrm{SD}=$ standard deviation. *Except where noted otherwise.

$\dagger$ A single dose of acetaminophen or ibuprofen was administered before the emergency department visit and at least 6 hours before the intervention. Ketorolac was administered Intravenously in the emergency department 2.8 to 4.3 hours before the intervention in all cases.

tOndansetron in all cases.

§Pantoprazole or orally administered mixture of bismuth salicylate and viscous lidocaine. ฯSome participants had more than 1 comorbidity; diagnoses were obtained by self-report and from the medical record.

\section{Results}

Of 4818 children screened, 236 were randomly allocated to receive hyoscine butylbromide $(n=120)$ or acetaminophen $(n=116)$ (Figure 1). The follow-up survey was completed by $73 \%$ of participants in both groups. Overall, the mean age was 12.4 (SD 3) years, and 153 participants (64.8\%) were girls. One-third of participants in both groups had received analgesia before enrolment (Table 1). Among the 212 children who were eligible but declined consent, the mean age was 11.9 (SD 2.9) years, and 129 (60.8\%) were girls.

\section{Primary outcome}

Four participants in the hyoscine butylbromide group and 1 participant in the acetaminophen group did not complete the 80-minute pain assessment. The mean pain scores before the intervention were $60 \mathrm{~mm}$ (SD $18 \mathrm{~mm}$ ) and $62 \mathrm{~mm}$ (SD $17 \mathrm{~mm}$ ) in the hyoscine butylbromide and acetaminophen groups, respectively. The 
Table 2: Mean pain scores*

Mean score $\pm \mathrm{SD}, \mathrm{mm}$

Time

Before intervention

After intervention

$15 \mathrm{~min}$

$30 \mathrm{~min}$

$45 \mathrm{~min}$

$60 \mathrm{~min}$

$80 \mathrm{~min}$
Hyoscine butylbromide $n=120$

$60.3 \pm 17.9$

$45.9 \pm 22.5$

$42.1 \pm 22.9$

$37.1 \pm 24.4$

$33.4 \pm 26.4$

$29.4 \pm 26.4$
Acetaminophen $n=116$

$62.3 \pm 16.5$

$45.5 \pm 23.8$

$39.3 \pm 24.1$

$36.7 \pm 26.8$

$33.7 \pm 27.9$

$30.1 \pm 28.8$
Adjusted difference $(95 \% \mathrm{Cl}) \dagger$

Note: $\mathrm{Cl}=$ confidence interval, $\mathrm{SD}=$ standard deviation

${ }^{*}$ Assessed with a $100 \mathrm{~mm}$ visual analogue scale.

tRepresents between-group difference in pain scores 80 minutes after the intervention, adjusted for preintervention pain scores.

corresponding scores at 80 minutes were $29 \mathrm{~mm}$ (SD $26 \mathrm{~mm}$ ) and $30 \mathrm{~mm}$ (SD $29 \mathrm{~mm}$ ), with an adjusted between-group difference of 1 (95\% confidence interval -7 to 7 ) (Table 2). There was no significant effect of any preintervention analgesia ( $p=0.9)$, or acetaminophen $(p=0.8)$, ibuprofen $(p=0.5)$ or ketorolac $(p=0.99)$ specifically, upon the results of the primary analysis.

\section{Secondary outcomes}

Rescue analgesia was administered to 4 participants (3.3\%) in the hyoscine butylbromide and 1 participant $(0.9 \%)$ in the acetaminophen group $(p=0.2)$. In all cases, ibuprofen or ketorolac was administered after the 80-minute pain assessment. A pain score less than $30 \mathrm{~mm} 80$ minutes after the intervention was reported by 66 participants $(55.0 \%)$ in the hyoscine butylbromide group and 63 participants $(54.3 \%)$ in the acetaminophen group $(p=0.9)$.

\section{Adverse effects}

Adverse effects in the emergency department were reported by $32 / 116(27.6 \%)$ and $28 / 115$ (24.3\%) participants in the hyoscine butylbromide and acetaminophen groups, respectively $(p=0.5)$ (Table 3). There were no serious adverse effects or missed surgical diagnoses.

\section{Other outcomes}

Caregiver satisfaction was high with both hyoscine butylbromide (median Likert score 5 [IQR 4 to 5]) and acetaminophen (median score 5 [IQR 3 to 5$]$ ). The mean visual analogue scale pain scores declined steadily after the intervention in both groups (Table 2). The median time to $20 \%$ reduction in preintervention pain score was 22.5 (IQR 15 to 60 ) minutes in the hyoscine butylbromide and 30.0 (IQR 15 to 60 ) minutes in the acetaminophen group (Figure 2). The median length of emergency department stay was 230.5 (IQR 189.8 to 292.3) minutes in the hyoscine butylbromide group and 236.0 (IQR 191.3 to 291.0) minutes in the acetaminophen group. Most participants in both groups were discharged from the emergency department, and few returned to a health care provider for abdominal pain (Table 4). Pain after discharge was reported by $46 / 84$ $(54.8 \%)$ of participants in the hyoscine butylbromide and $41 / 84$ $(48.8 \%)$ of those in the acetaminophen group.
Table 3: Adverse effects in the emergency department and at 72-hour follow-up

\section{No. $(\%)$ of patients}

\begin{tabular}{|c|c|c|c|}
\hline Adverse effect & $\begin{array}{c}\text { Hyoscine } \\
\text { butylbromide }\end{array}$ & Acetaminophen & $p$ value \\
\hline $\begin{array}{l}\text { In emergency } \\
\text { department }\end{array}$ & $n=116^{\star}$ & $n=115^{\star}$ & \\
\hline Any adverse effect $\dagger$ & $32(27.6)$ & $28(24.3)$ & 0.5 \\
\hline Nausea & $10(8.6)$ & $12(10.4)$ & \\
\hline Dizziness & 15 (12.9) & $8(7.0)$ & \\
\hline Dry mouth & $5(4.3)$ & $7(6.1)$ & \\
\hline Photosensitivity & $9(7.8)$ & $3(2.6)$ & \\
\hline Vomiting & $3(2.6)$ & $1(0.9)$ & \\
\hline Constipation & $1(0.9)$ & $3(2.6)$ & \\
\hline Dry skin & $2(1.7)$ & $2(1.7)$ & \\
\hline Racing heart & $2(1.7)$ & $2(1.7)$ & \\
\hline Headache & $1(0.9)$ & $2(1.7)$ & \\
\hline Diarrhea & $0(0.0)$ & $1(0.9)$ & \\
\hline Sweating & $0(0.0)$ & $1(0.9)$ & \\
\hline Drowsiness & $1(0.9)$ & $0(0.0)$ & \\
\hline 72-h follow-up & $n=84 \ddagger$ & $n=84 \ddagger$ & \\
\hline Any adverse effect $\dagger$ & $12(14.3)$ & $12(14.3)$ & 0.99 \\
\hline Dry mouth & $3(3.6)$ & $4(4.8)$ & \\
\hline Constipation & $0(0.0)$ & $1(1.2)$ & \\
\hline Vomiting & $0(0.0)$ & $2(2.4)$ & \\
\hline Dizziness & $4(4.8)$ & $0(0.0)$ & \\
\hline Light sensitivity & $1(1.2)$ & $1(1.2)$ & \\
\hline Hives & $0(0.0)$ & $1(1.2)$ & \\
\hline Drowsiness & $4(4.8)$ & $2(2.4)$ & \\
\hline Headache & $2(2.4)$ & $2(2.4)$ & \\
\hline \multicolumn{4}{|c|}{$\begin{array}{l}\text { *Denominator reflects the number of participants who completed the } 80 \text {-minute pain score. } \\
\text { †Some participants reported more than } 1 \text { adverse effect. } \\
\ddagger \text { Denominator reflects the number of participants for whom telephone follow-up alone } \\
\text { was performed. }\end{array}$} \\
\hline
\end{tabular}




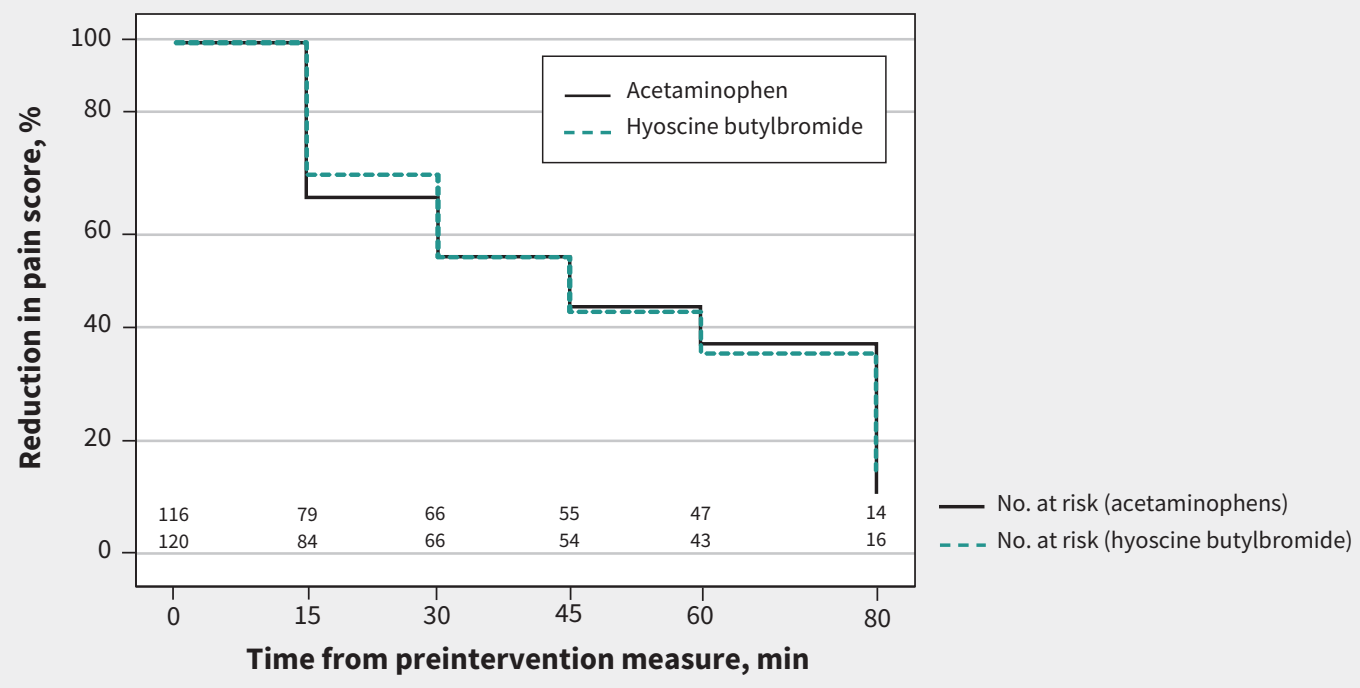

Figure 2: Kaplan-Meier survival analysis, showing time to reduction in visual analogue scale pain score after intervention.

Table 4: Discharge diagnoses, disposition from emergency department and follow-up

\section{No. $(\%)$ of participants}

\section{Hyoscine}

butylbromide Acetaminophen

Variable $n=116$ $n=115$

\section{Discharge diagnosis*}

Abdominal pain not yet

$77(66.4)$

$77(67.0)$

determined

Gastroenteritis

37 (31.9)

Functional abdominal pain

Irritable bowel syndrome

$2(1.7)$

\section{Disposition}

Admitted $\dagger$

Discharged

\section{2-h follow-upł}

Returned to medical care for

$$
6(5.2)
$$

abdominal pain

Hospital admission

Ongoing abdominal pain§

*Based largely on physician judgment.

†Admitted by the pediatric general surgery service for observation.

$\ddagger$ Data obtained via telephone survey; for participants who could not be reached by telephone, data were obtained from the electronic medical record and therefore reflect the limits of the system's catchment area.

$\S$ Telephone survey alone.

\section{Interpretation}

In this randomized controlled trial, we found that hyoscine butylbromide was not superior to acetaminophen in children with nonspecific abdominal pain in the highly selected patients randomized. Our findings suggest that either agent may be routinely considered for children with nonspecific colicky abdominal pain in the emergency department. Among adults, a reduction of $30 \mathrm{~mm}$ or more on a visual analogue scale corresponds to "adequate pain control," decrease of $30 \%$ or more from baseline has been deemed clinically significant in irritable bowel syndrome. ${ }^{48}$ We observed a decrease from baseline of roughly $50 \%$ in both groups, but this was achieved by less than $60 \%$ of participants. However, caregiver satisfaction was high in both groups. Pain severity at discharge and satisfaction with pediatric emergency department care are poorly correlated, ${ }^{49,50}$ and caregiver satisfaction may reflect a "personal evaluation of health care services and providers," ${ }^{\prime 51}$ along with the message that pain management is a priority. ${ }^{49,52}$ Ongoing pain after discharge was reported by about half of participants in both groups, which shows the need for appropriate discharge instructions regarding analgesia. More than half of participants in both groups received analgesia before enrolment. The possibility of a residual analgesic effect complementing that of the intervention was unlikely because we excluded participants who had received analgesia within 6 hours, the therapeutic window of both hyoscine butylbromide and acetaminophen.

Our findings are in keeping with those of adult studies of orally administered hyoscine butylbromide for colicky abdominal pain that showed decreases in pain of $59 \%{ }^{53}$ and $30 \% .^{54}$ In the only known pediatric study of hyoscine butylbromide, the medication was compared to Spascupreel (Biologische Heilmittel Heel), a homeopathic 
preparation, in children with recurrent gastrointestinal or urethral spasms; the study reported that both agents were beneficial, with few adverse effects. ${ }^{30}$ Although hyoscine butylbromide is an antimuscarinic agent, the butylbromide moiety limits systemic absorption, and, therefore, systemic anticholinergic effects are uncommon. ${ }^{28,29,46}$ To our knowledge, no pediatric studies have explored acetaminophen for nonspecific abdominal pain. Remington-Hobbs and colleagues ${ }^{46}$ found that, in adults, acetaminophen was superior to intravenously given hyoscine butylbromide for "undifferentiated" abdominal pain. Hyoscine butylbromide is available in Canadian emergency departments but must be ingested as an intact pill. Acetaminophen may be a more feasible option because it is available over the counter and inexpensive, and can be administered to children of all ages. Adopting a therapy with a time to effective analgesia of $60-80$ minutes postintervention may be difficult in an acute care setting. In our study, the median length of stay was more than 3.5 hours in both groups, consistent with data from a US cohort of children with "undifferentiated" abdominal pain (3.4 h). ${ }^{5}$ We did not record the number of diagnostic tests, but tests may have contributed to length of stay, ${ }^{55,56}$ and triagebased directives may facilitate more timely analgesia and discharge. ${ }^{57}$

Future studies should explore the effectiveness of a higher dosage of hyoscine butylbromide $(20 \mathrm{mg}$ ), hyoscine patches or combining pharmacologic therapies with nonpharmacologic strategies such as cognitive behaviour therapy. ${ }^{58}$ Where possible, should ethical approval allow, studies could include a placebo arm to test whether an active comparator is associated with earlier symptom resolution and whether the benefits of therapy offset the costs.

\section{Limitations}

The observed decreases in pain in our study may have been due to the study medications, the natural history of nonspecific abdominal pain, ${ }^{59}$ satisfaction that diagnostic investigations were negative or "tincture of time." Definitively attributing analgesia to the intervention would have been possible with a placebo arm. Nevertheless, it remains likely that participants in both groups benefited from the interventions. Following pain duration of roughly 2 hours before enrolment, pain scores had decreased by about $50 \%$ in both groups 60-80 minutes after the intervention, the time of peak analgesic effectiveness of both agents..$^{19,34,35}$ In adult emergency department patients, intravenously administered hyoscine butylbromide and orally administered acetaminophen were associated with decreases greater than $50 \%$ in "undifferentiated" abdominal pain at 60 minutes. ${ }^{46}$ In the present study, many screened patients were excluded because of suspected underlying causes of the abdominal pain, which may limit external generalizability. We focused on nonspecific pain because it is the most common form of abdominal pain among children presenting to the emergency department. ${ }^{8,9}$ We limited enrolment to children with colicky pain because we believed it was amenable to relief with hyoscine butylbromide, an inhibitor of acetylcholine-mediated intestinal smooth muscle contraction. ${ }^{19}$ Furthermore, we limited enrolment to patients able to verbalize symptoms consistent with colicky abdominal pain; thus, our findings may not apply to patients with other types of abdominal pain. Although we were able to determine return visits for almost all participants, for roughly a quarter, we could not determine whether they had persistent pain or delayed adverse effects.

\section{Conclusion}

Hyoscine butylbromide was not superior to acetaminophen in children with nonspecific colicky abdominal pain, but both were associated with a clinically important benefit. Our results suggest that either hyoscine butylbromide or acetaminophen can be considered for children with nonspecific colicky abdominal pain, the latter being more practical. Definitive recommendations require a placebo-controlled trial to determine whether the benefits of providing analgesia are clinically important compared to no analgesia with respect to outcomes such as emergency department length of stay, satisfaction and side effects.

\section{References}

1. Saps M, Seshadri R, Schaffer G, et al. A prospective school-based study of abdominal pain and other common somatic complaints in children. J Pediatr 2009;154:322-6.

2. Niska R, Bhuijya F, Xu J. National Hospital Ambulatory Medical Care Survey: 2007 emergency department summary. Natl Health Stat Report 2010;6:1-31.

3. Alpern ER, Stanley RM, Gorelick MH, et al.; Pediatric Emergency Care Applied Research Network. Epidemiology of a pediatric emergency medicine research network: the PECARN Core Data Project. Pediatr Emerg Care 2006;22:689-99.

4. Loening-Baucke V, Swidsinski A. Constipation as cause of acute abdominal pain in children. J Pediatr 2007;151:666-9.

5. Harris BR, Chinta SS, Colvin R, et al. Undifferentiated abdominal pain in children presenting to the pediatric emergency department. Clin Pediatr (Phila) 2019;58:1212-23.

6. Poonai N, Cowie A, Davidson C, et al. Reported provision of analgesia to patients with acute abdominal pain in Canadian paediatric emergency departments. CJEM 2016;18:323-30.

7. Poonai N, Paskar D, Konrad SL, et al. Opioid analgesia for acute abdominal pain in children: a systematic review and meta-analysis. Acad Emerg Med 2014;21:1183-92.

8. Falch $\mathrm{C}$, Vicente $\mathrm{D}$, Häberle $\mathrm{H}$, et al. Treatment of acute abdominal pain in the emergency room: a systematic review of the literature. Eur J Pain 2014;18:902-13.

9. Viniol A, Keunecke C, Biroga T, et al. Studies of the symptom abdominal pain a systematic review and meta-analysis. Fam Pract 2014;31:517-29.

10. Friedrichsdorf SJ, Postier A, Eull D, et al. Pain outcomes in a US children's hospital: a prospective cross-sectional survey. Hosp Pediatr 2015;5:18-26.

11. Perrott DA, Piira T, Goodenough B, et al. Efficacy and safety of acetaminophen vs ibuprofen for treating children's pain or fever: a meta-analysis. Arch Pediatr Adolesc Med 2004;158:521-6.

12. Sjoukes A, Venekamp RP, van de Pol AC, et al. Paracetamol (acetaminophen) or non-steroidal anti-inflammatory drugs, alone or combined, for pain relief in acute otitis media in children. Cochrane Database Syst Rev 2016;(12):CD011534.

13. Cooper TE, Fisher E, Anderson B, et al. Paracetamol (acetaminophen) for chronic non-cancer pain in children and adolescents. Cochrane Database Syst Rev 2017;(8):CD012539.

14. Eccleston C, Cooper TE, Fisher E, et al. Non-steroidal anti-inflammatory drugs (NSAIDs) for chronic non-cancer pain in children and adolescents. Cochrane Database Syst Rev 2017;(8):CD012537.

15. Fein JA, Zempsky WT, Cravero JP; Committee on Pediatric Emergency Medicine and Section on Anesthesiology and Pain Medicine, American Academy of Pediatrics. Relief of pain and anxiety in pediatric patients in emergency medical systems. Pediatrics 2012;130:e1391-405.

16. Herd DW, Babl FE, Gilhotra Y; PREDICT group. Pain management practices in paediatric emergency departments in Australia and New Zealand: a clinical and organizational audit by National Health and Medical Research Council's National Institute of Clinical Studies and Paediatric Research in Emergency Departments International Collaborative. Emerg Med Australas 2009;21:210-21.

17. Whiston $\mathrm{C}$, Ali S, Wright B, et al. Is caregiver refusal of analgesics a barrier to pediatric emergency pain management? A cross-sectional study in two Canadian centres. CJEM 2018;20:892-902.

18. Lee WH, O'Brien S, Skarin D, et al.; PREDICT. Pediatric abdominal pain in children presenting to the emergency department. Pediatr Emerg Care 2019 Mar. 12 [Epub ahead of print]. doi: 10.1097/PEC.0000000000001789.

19. Tytgat GN. Hyoscine butylbromide: a review of its use in the treatment of abdominal cramping and pain. Drugs 2007;67:1343-57.

20. De Gregorio M, Damiani S, Gatta G. Antalgic properties of proxazole. Double blind study in visceral algoplastic conditions. Panminerva Med 1969;11:436-40.

21. Navarro Martinez B. Statistical double-blind study on the therapeutic preference of chronic gastropathic subjects in acute crisis. Rev Med Suisse 1972;4:77-9.

22. Sieg H. Double blind test using hyoscine- $N$-butyl bromide (Buscopan) to reduce pain in ulcus ventriculi [article in German]. Z Gastroenterol 1974;12:235-8. 
23. Miyoshi A, Suyama T, Kawaura I. A double-blind comparative study of the inhibitory effect of intraduodenally administered hyoscine- $N$-butylbromide on human duodenal motility. J Int Med Res 1977;5:223-32.

24. Metzger KH. The effect of anticholinergic agents on caudal intestinal section: experimental investigations into motility [article in German]. Arztl Prax 1979;21:923-4.

25. Ritchie JA, Truelove SC. Treatment of irritable bowel syndrome with lorazepam, hyoscine butylbromide, and ispaghula husk. BMJ 1979;1:376-8.

26. Nigam P, Kapoor KK, Rastog CK, et al. Different therapeutic regimens in irritable bowel syndrome. J Assoc Physicians India 1984;32:1041-4.

27. Sánchez Martinez J, Goiz Durán I. Clinical assessment of the tolerability and the effect of IK-19 in tablet form on pain of spastic origin. Invest Med Int 1988;15:63-5.

28. Schäfer E, Ewe K. The treatment of irritable colon. Efficacy and tolerance of buscopan plus, buscopan, paracetamol and placebo in ambulatory patients with irritable colon [article in German]. Fortschr Med 1990;108:488-92.

29. Mueller-Lissner S, Tytgat GN, Paulo LG, et al. Placebo- and paracetamolcontrolled study on the efficacy and tolerability of hyoscine butylbromide in the treatment of patients with recurrent crampy abdominal pain. Aliment Pharmacol Ther 2006;23:1741-8.

30. Müller-Krampe B, Oberbaum M, Klein P, et al. Effects of Spascupreel versus hyoscine butylbromide for gastrointestinal cramps in children. Pediatr Int 2007;49:328-34.

31. Bieri D, Reeve RA, Champion CD, et al. The Faces Pain Scale for the self-assessment of the severity of pain experienced by children: development, initial validation, and preliminary investigation for ratio scale properties. Pain 1990;41:139-50.

32. Harris PA, Taylor R, Thielke R, et al. Research electronic data capture (REDCap) - a metadata-driven methodology and workflow process for providing translational research informatics support. J Biomed Inform 2009;42:377-81.

33. Humphrey GB, Boon CM, van Linden GF, et al. The occurrence of high levels of acute behavioral distress in children and adolescents undergoing routine venipunctures. Pediatrics 1992;90:87-91.

34. Kelley MT, Walson PD, Edge JH, et al. Pharmacokinetics and pharmacodynamics of ibuprofen isomers and acetaminophen in febrile children. Clin Pharmacol Ther 1992;52:181-9.

35. Anderson BJ. What we don't know about paracetamol in children. Paediatr Anaesth 1998;8:451-60.

36. Clark E, Plint AC, Correll R, et al. A randomized, controlled trial of acetaminophen, ibuprofen, and codeine for acute pain relief in children with musculoskeletal trauma. Pediatrics 2007;119:460-7.

37. Borland $\mathrm{M}$, Jacobs I, King B, et al. A randomized controlled trial comparing intranasal fentanyl to intravenous morphine for managing acute pain in children in the emergency department. Ann Emerg Med 2007;49:335-40.

38. Bird SB, Dickson EW. Clinically significant changes in pain along the visual analog scale. Ann Emerg Med 2001;38:639-43.

39. Gallagher EJ, Liebman M, Bijur PE. Prospective validation of clinically important changes in pain severity measured on a visual analog scale. Ann Emerg Med 2001;38:633-8.

40. Kelly AM. Does the clinically significant difference in visual analog scale pain scores vary with gender, age or cause of pain? Acad Emerg Med 1998;5:1086-90.

41. Kelly AM. The minimum clinically significant difference in the visual analogue scale pain score does not differ with severity of pain. Emerg Med J 2001;18:205-7.
42. von Baeyer CL, Spagrud LJ, McCormick JC, et al. Three new datasets supporting use of the Numerical Rating Scale (NRS-11) for children's self-reports of pain intensity. Pain 2009;143:223-7.

43. Powell CV, Kelly AM, Williams A. Determining the minimum clinically significant difference in visual analog pain score for children. Ann Emerg Med 2001;37:28-31.

44. Jadad AR, Browman GP. The WHO analgesic ladder for cancer pain management. Stepping up the quality of its evaluation. JAMA 1995;274:1870-3.

45. Todd KH, Funk KG, Funk JP, et al. Clinical significance of reported changes in pain severity. Ann Emerg Med 1996;27:485-9.

46. Remington-Hobbs J, Petts G, Harris T. Emergency department management of undifferentiated abdominal pain with hyoscine butylbromide and paracetamol: a randomised control trial. Emerg Med J 2012;29:989-94.

47. Lee JS, Hobden E, Stiell IG, et al. Clinically important change in the visual analog scale after adequate pain control. Acad Emerg Med 2003;10:1128-30.

48. Irritable bowel syndrome - clinical evaluation of drugs for treatment. Silver Spring (MD): US Food and Drug Administration; 2012, updated 2018 Aug. 24. Available: www.fda.gov/regulatory-information/search-fda-guidance-documents/irritable -bowel-syndrome-clinical-evaluation-products-treatment (accessed 2020 Feb. 14).

49. Ali S, Weingarten LE, Kircher J, et al. A survey of caregiver perspectives on children's pain management in the emergency department. CJEM 2016;18:98-105.

50. Magaret ND, Clark TA, Warden CR, et al. Patient satisfaction in the emergency department - a survey of pediatric patients and their parents. Acad Emerg Med 2002;9:1379-88.

51. Kelly AM. Patient satisfaction with pain management does not correlate with initial or discharge VAS pain score, verbal pain rating at discharge, or change in VAS score in the emergency department. J Emerg Med 2000;19:113-6.

52. Ward SE, Gordon D. Application of the American Pain Society quality assurance standards. Pain 1994;56:299-306.

53. Ge Z, Yuan Y, Zhang S, et al. Efficacy and tolerability of two oral hyoscine butylbromide formulations in Chinese patients with recurrent episodes of self-reported gastric or intestinal spasm-like pain. Int J Clin Pharmacol Ther 2011; 49:198-205.

54. Lacy BE, Wang F, Bhowal S, et al. On-demand hyoscine butylbromide for the treatment of self-reported functional cramping abdominal pain. Scand J Gastroenterol 2013;48:926-35.

55. Lee J, Kirschner J, Pawa S, et al. Computed tomography use in the adult emergency department of an academic urban hospital from 2001 to 2007. Ann Emerg Med 2010;56:591-6.

56. Kirschner J, Shah K, Runde D, et al. Patterns in computed tomography utilization among emergency physicians in an urban, academic emergency department. Emerg Radiol 2014;21:577-81.

57. Ender KL, Krajewski JA, Babineau J, et al. Use of a clinical pathway to improve the acute management of vaso-occlusive crisis pain in pediatric sickle cell disease. Pediatr Blood Cancer 2014;61:693-6.

58. Abbott RA, Martin AE, Newlove-Delgado TV, et al. Psychosocial interventions for recurrent abdominal pain in childhood. Cochrane Database Syst Rev 2017; (1):CD010971.

59. Rasquin A, Di Lorenzo C, Forbes D, et al. Childhood functional gastrointestinal disorders: child/adolescent. Gastroenterology 2006;130:1527-37.

\section{Competing interests: None declared.}

This article has been peer reviewed.

Affiliations: Division of Emergency Medicine (Poonai, Kumar, Coriolano, Brahmbhatt, Dzongowski, Stevens, Gupta, Miller, Elsie, Joubert, Lim), Department of Paediatrics, and Departments of Internal Medicine (Poonai, Elsie) and Epidemiology and Biostatistics (Poonai, Elsie), Schulich School of Medicine and Dentistry, Western University; Children's Health Research Institute (Poonai, Miller, Lim), London Health Sciences Centre, London, Ont.; Departments of Pediatrics (Thompson) and Emergency Medicine (Thompson), Cumming School of Medicine, and Alberta Children's Hospital Research Institute (Thompson), University of Calgary, Calgary, Alta.; Division of Gastroenterology (Ashok), Department of Paediatrics, Schulich School of Medicine and Dentistry,
Western University; Division of Paediatric Surgery (Bütter), Department of Surgery, Schulich School of Medicine and Dentistry, Western University, London, Ont.; Department of Pediatrics (Ali), Faculty of Medicine \& Dentistry, and Women and Children's Health Research Institute (Ali), University of Alberta, Edmonton, Alta.

Contributors: Naveen Poonai, Samina Ali and Gary Joubert conceptualized and designed the study, with contributions from Priti Gupta and Holly Stevens, and drafted the manuscript. Kriti Kumar, Shaily Brahmbhatt, Sharlene Elsie and Emily Dzongowski assisted with data collection. Michael Miller analyzed the data. Kriti Kumar, Kamary Coriolano, Shaily Brahmbhatt, Sharlene Elsie and Emily Dzongowski interpreted the data. Naveen Poonai, Samina Ali, Michael Miller, Graham Thompson, Rod Lim, Andreana Bütter,
Kamary Coriolano and Dhandapani Ashok revised the manuscript critically for important intellectual content. All of the authors approved the final version to be published and agreed to be accountable for all aspects of the work.

Funding: This study was supported by the Lawson Health Research Institute Internal Research Fund. The sponsors had no role in the conduct of the trial, interpretation of the data or presentation of results.

Data sharing: All portions of the data are available to others if the data are intended to be used for scholarly purposes such as a systematic review. Data may be accessed by emailing the corresponding author.

Accepted: July 27, 2020

Correspondence to: Naveen Poonai, naveen.poonai@lhsc.on.ca 